\title{
B-Cell Targeted Treatments for Neuromyelitis Optica Spectrum Disorder: A Focus on CDI9 and CD20
}

\author{
Michael Levy' \\ Maureen A Mealy iD $^{2}$ \\ 'Department of Neurology, \\ Massachusetts General Hospital \& \\ Harvard Medical School, Boston, MA, \\ USA; ${ }^{2}$ Medical Affairs, Horizon \\ Therapeutics, Deerfield, IL, USA
}

\begin{abstract}
Neuromyelitis optica spectrum disorder (NMOSD) is a rare relapsing autoimmune disease of the central nervous system that preferentially targets the optic nerves and spinal cord, leading to visual loss and impaired mobility. Until 2019, no medications were FDA-approved for NMOSD treatment, and standard of care was based on mostly empiric and retrospective data. Therapies that target B cells emerged as a treatment strategy due to their fundamental role in disease pathogenesis. We explore different monoclonal antibodies directed at either CD20+ or CD19+ B cells that may have utilization in the treatment of NMOSD, discussing what is known regarding their efficacy and safety.
\end{abstract}

Keywords: rituximab, inebilizumab, NMO, MOG, monoclonal

\section{Introduction}

Neuromyelitis optica spectrum disorder (NMOSD) is a rare autoimmune disease characterized by recurrent inflammatory attacks of the central nervous system (CNS) with a predilection for the optic nerves and spinal cord. ${ }^{1}$ Antibodies against the water channel protein aquaporin 4 (AQP4-Ab) can be detected in $\sim 80 \%$ of patients. ${ }^{2} \mathrm{AQP} 4-$ Abs have been proposed to be pathogenic when they access the CNS and bind AQP4 on the end-feet of astrocytes, thus stimulating a cascade of inflammatory events that lead to axonal, neuronal and myelin damage in NMOSD. ${ }^{3}$ Symptoms during acute attacks correspond to the structures within the CNS that are inflamed, and can include loss of vision, marked extremity weakness, and altered sensation. ${ }^{1}$ Attacks are often severe, recovery is rarely complete, and attack prevention is paramount to delaying the accrual of disability over time. ${ }^{4}$ Until 2019, no medications were FDA-approved for NMOSD treatment, and standard of care was based on mostly empiric and retrospective off-label use of several immunomodulatory medications, including rituximab, a chimeric monoclonal antibody (mAb) that targets CD20+ B cells. ${ }^{5}$ Data support that B cells play a complex and fundamental role in the pathogenesis of NMOSD through several mechanisms, including pathogenic AQP4 antibody production and secretion by plasmablasts and plasma cells, proinflammatory cytokine secretion, and antigen presentation (Figure 1). ${ }^{3}$ We explore B cell targeted strategies that have been utilized in NMOSD and discuss what is known regarding their efficacy and safety (Table 1).

\section{CD20-Directed Therapies in NMOSD}

\section{Rituximab}

Rituximab was first approved in 1997 for non-Hodgkin's lymphoma and subsequently received an indication for chronic lymphocytic leukemia and rheumatoid 




Figure I B cells migrate from from the peripheral circulation to the central nervous system after they are activated in lymph nodes. AQP4 antibodies are mostly produced by peripheral AQP4-reactive $B$ cells but during a relapse, some plasmablasts make AQP4 antibody within the CNS.

Notes: Reproduced from: Kowarik MC, Astling D, Gasperi C, et al. CNS Aquaporin-4-specific B cells connect with multiple B-cell compartments in neuromyelitis optica spectrum disorder. Annals of Clinical and Translational Neurology. 2017; 4(6): 369-380. ${ }^{3}$ With permission from Dr. Bennett. Copyright (c 2017 The Authors. Creative Commons Attribution-NonCommercial-NoDerivs (https://creati vecommons.org/licenses/by-nc-nd/3.0/nz/legalcode).

arthritis. $^{6}$ Its off-label use has been reported in many additional indications, including NMOSD and multiple sclerosis (MS). ${ }^{7,8}$ The CD20+ B cells targeted by rituximab include cell lineage from pre-B through mature B cells, primarily through complement-dependent cytotoxicity (CDC). ${ }^{9}$ Because the antibody-producing mature plasma cells do not express CD20, this suggests that rituximab primarily works through antibody-independent mechanisms. ${ }^{10}$ Targeting B cells with rituximab emerged following an investigation by Cree et al in 2005, in which a marked reduction in relapses was achieved following rituximab treatment for a mean 12-month follow-up among eight patients who fulfilled the 1999 NMO diagnostic criteria. ${ }^{11}$ These promising results prompted further investigation through over 20 retrospective and prospective studies around the globe with mostly favorable data, ${ }^{8}$ including two randomized controlled trials (RCT), ${ }^{12,13}$ of which one was placebo-controlled and blinded (RIN-1, $\mathrm{n}=19$ per arm) ${ }^{13}$

As these data continued to accumulate, the standard of care for NMOSD treatment began to shift such that rituximab was increasingly used as first-line therapy, despite the lack of an adequately powered and rigorously conducted registrational trial that might lend itself to regulatory approval for this disease. Data from a recent meta-analysis examined the mean annualized relapse rate (ARR) among 577 patients from 26 studies, which resulted in a mean 1.56 (95\% CI, -1.82 to -1.29 ) reduction in the mean ARR. ${ }^{14}$ A second meta-analysis among 438 patients from 46 studies calculated a mean $0.79(95 \% \mathrm{CI},-1.08$ to -0.49$)$ reduction in the mean ARR. ${ }^{8}$ Both analyses acknowledge the heterogeneity among studies, limiting the interpretation of these pooled data sets. The single blinded placebo-controlled trial conducted in NMOSD, RIN-1, included 19 participants per arm. ${ }^{13}$ Sixteen of 19 participants assigned to receive rituximab completed the study, none of whom relapsed during the 72-week follow-up, whereas 7 (37\%) of participants receiving placebo relapsed. While the study is limited by its small sample size and inclusion of participants with mild disease in a homogeneous patient cohort, it nonetheless represents the highest class of data available for rituximab treatment in NMOSD.

The longevity of rituximab use across many indications allows for a relatively well-known safety profile. Infusion reactions are among the most reported adverse events, with between 32 and $77 \%$ of patients reporting a reaction to the initial dose and less reactivity to subsequent doses. ${ }^{6}$ However, premedication to mitigate the risk of infusion reactions was recommended only after initial approval, and the lack of these medications in earlier trials may have contributed to the higher rates of infusion reactions. Premedication with corticosteroids, acetaminophen and diphenhydramine has become the standard of care in NMOSD. ${ }^{5}$ In RIN-1, participants were premedicated with acetaminophen and diphenhydramine, and were concomitantly on a small dose of an oral corticosteroid. ${ }^{13}$ Thirty-seven percent of participants experienced an infusion reaction compared to none who received placebo, though none were serious. Importantly, rituximab carries a boxed warning associated with fatal infusion reactions, most often within 24 hours of the initial dose. ${ }^{6}$ Other boxed warnings identified in the label include tumor lysis syndrome (which would presumably not be applicable to the NMOSD population), severe mucocutaneous reactions (some fatal), and progressive multifocal leukoencephalopathy (PML; some fatal). However, while there is no formal tracking, no such fatalities have been reported in the NMOSD population linked to rituximab treatment. Infections, sometimes serious, are also associated with rituximab, including reactivation of hepatitis $\mathrm{B}^{6}{ }^{6}$ In NMOSD, no safety signal related to infections emerged during the RIN-1 trial, beyond what is known regarding rituximab use overall. ${ }^{13}$ 
Table I Summary of B Cell Targeted Treatments for NMOSD

\begin{tabular}{|c|c|c|c|c|c|}
\hline & Rituximab & Ocrelizumab & Ofatumumab & Ublituximab & Inebilizumab \\
\hline Structure & Chimeric IgGI* & $\begin{array}{l}\text { Humanized } \\
\operatorname{lgGI}\end{array}$ & $\begin{array}{l}\text { Fully human } \\
\text { lgGI }\end{array}$ & $\begin{array}{l}\text { Glycoengineered } \\
\text { chimeric } \lg G \mid\end{array}$ & $\begin{array}{l}\text { Glycoengineered humanized } \\
\text { lgGI }\end{array}$ \\
\hline Target & CD20+ B cells & CD20+ B cells & CD20+ B cells & CD20+ B cells & CDI9+ B cells \\
\hline Primary MOA & CDC* & ADCC* & CDC & $A D C C$ & $A D C C$ \\
\hline $\begin{array}{l}\text { Boxed } \\
\text { warnings }\end{array}$ & $\begin{array}{l}\text { - Fatal infusion reactions } \\
\text { - Tumor lysis syndrome } \\
\text { - Severe mucocutaneous } \\
\text { reactions } \\
\text { - PML* }\end{array}$ & None & None & None & None \\
\hline $\begin{array}{l}\text { Effectiveness } \\
\text { data in } \\
\text { NMOSD }\end{array}$ & $\begin{array}{l}\text { - }>20 \text { open-label and } \\
\text { retrospective analyses } \\
\text { - Open-label RCT* } \\
\text { - Placebo-controlled and } \\
\text { blinded RCT (RIN-I; } \mathrm{n}=38 \text { ) }\end{array}$ & $\begin{array}{l}\text { None } \\
\text { reported }\end{array}$ & Case report & $\begin{array}{l}\text { Open-label acute } \\
\text { treatment trial } \\
(n=5)\end{array}$ & $\begin{array}{l}\text { Placebo-controlled and blinded } \\
\text { RCT }(n=230)\end{array}$ \\
\hline $\begin{array}{l}\text { Dosing } \\
\text { Regimen in } \\
\text { NMOSD }\end{array}$ & Not standardized & $\begin{array}{l}\text { No data in } \\
\text { indication }\end{array}$ & $\begin{array}{l}\text { Limited/no data } \\
\text { in indication }\end{array}$ & $\begin{array}{l}\text { Limited/no data in } \\
\text { indication }\end{array}$ & $\begin{array}{l}300 \mathrm{mg} \text { IV q6 months, following } \\
2 \text { paired doses at initiation }\end{array}$ \\
\hline $\begin{array}{l}\text { Infusion } \\
\text { reactions in } \\
\text { NMOSD }\end{array}$ & $\begin{array}{l}37 \% \text { in RIN-I (none serious), } \\
\text { compared to } 0 \% \text { on placebo }\end{array}$ & No data & No data & No data & $\begin{array}{l}9 \% \text {; comparable to placebo in } \\
\text { rate and severity }\end{array}$ \\
\hline $\begin{array}{l}\text { FDA-approval } \\
\text { for NMOSD }\end{array}$ & No & No & No & No & Yes \\
\hline
\end{tabular}

Abbreviations: *IgGI, immunoglobulin-I; CDC, complement-dependent cytotoxicity; ADCC, antibody-dependent cellular cytotoxicity; PML, progressive multifocal leukoencephalopathy; RCT, randomized-controlled trial.

Different dosing regimens were studied and approved for different indications. ${ }^{6}$ Given the lack of approval in NMOSD, dosing of this medication has not been standardized. Among the clinical investigations done in this disease, rituximab dose and dose timing ranged considerably, both within and between studies, ${ }^{8,15,16}$ and may influence response to treatment. In general, infusions run over at least four hours with rate escalation every thirty minutes up to the maximum dose, and infusions are interrupted or rates are reduced in response to infusion reactions.

\section{Ocrelizumab}

Ocrelizumab is another $\mathrm{mAb}$ that targets $\mathrm{CD} 20+\mathrm{B}$ cells, and it became the first $\mathrm{B}$ cell targeted monoclonal approved for MS in 2017. ${ }^{17}$ Several factors differentiate it from rituximab: it is a humanized antibody that targets a different antigenic target on CD20 which overlaps with the target-binding site of rituximab, and the primary mechanism of B cell depletion is through antibodydependent cellular cytotoxicity (ADCC), rather than
CDC. ${ }^{9}$ While there exists nothing in the literature regarding its use in NMOSD, its mechanism of action suggests it may be useful.

In MS clinical trials, the incidence of infusion reactions was $34-40 \%$, with most reactions experienced on first dose, despite the recommended premedication regimen, which looks similar to that currently used for rituximab. There were no fatal infusion reactions, but $0.3 \%$ were graded as serious, possibly requiring hospitalization. ${ }^{17}$ Like rituximab and owing to the mechanism of action of B cell depletion, infections are more frequently experienced by patients on ocrelizumab, and include an increased risk for upper and lower respiratory tract infections, skin infections, and herpes-related infections. However, there was no increased risk of serious infections. ${ }^{17}$ While not seen in the trials, PML has been reported post-marketing, including one patient with no previous exposure to other immunotherapies. ${ }^{18,19}$ Cancers, including breast cancer, were seen more frequently in trial patients treated with ocrelizumab and 
indicated a possible signal for increased risk, however, post-marketing data has not supported this concern. ${ }^{20}$ An analysis of the FDA Adverse Event Reporting Database noted that ocrelizumab was associated with an almost two times higher frequency of infections than rituximab. ${ }^{21}$ The authors speculated that this may point to a more extensive B cell depletion with ocrelizumab.

The dosing regimen for ocrelizumab was standardized for an MS indication: 2 paired infusions at onset, with half the standard dose delivered with each infusion, followed by a single full-dose infusion every 6 months. ${ }^{17}$ Infusion time is quicker with ocrelizumab than with its predecessor, such that the infusion can be delivered over as little as 2 hours, and infusions are interrupted or rates are reduced in response to infusion reactions.

\section{Ofatumumab}

Ofatumumab was the third anti-CD20 mAb, developed for MS and approved in 2020 for relapsing MS indication. ${ }^{22}$ It differentiates itself from its predecessors in two distinct ways: it is a fully human molecule and is delivered by $20 \mathrm{mg}$ subcutaneous injection (monthly, following an initial series of weekly injections x 3 weeks). ${ }^{9}$ The subcutaneous administration reduces the risk of infusion/injection-related reactions, without the need for premedication.

Two pivotal clinical trials that supported its approval for MS compared ofatumumab to an active comparator, teriflunomide. $^{23}$ The adverse event profiles looked similar between these two immunomodulatory therapies, with similar infection rates and no opportunistic infections observed during ofatumumab treatment. Injection-related reactions are significantly higher across all trials, compared to patients receiving placebo injections. ${ }^{22}$ They were generally mild to moderate in severity, and included fever, headache, myalgia, chills, and fatigue. Given the relatively recent approval, the healthcare community will continue to learn more regarding safety with increased post-marketing exposure. A case report in NMOSD describes a pediatric-onset AQP4-AB seropositive patient with highly active disease, in whom other immunotherapies failed. ${ }^{24}$ Most recent previous therapy was rituximab, which caused severe infusion reactions, including an anaphylactic-like reaction requiring hospitalization, and severe catheter-related sepsis. In addition, clinical relapses continued in the presence of a persistent B cell population. These factors led to the treatment change to ofatumumab. In the two years of follow-up on ofatumumab, the patient experienced no additional relapses and tolerated the medication well.

\section{Ublituximab}

The fourth is ublituximab, a glycoengineered anti-CD20 $\mathrm{mAb}$ currently being investigated for several indications, including MS, and is not currently approved for use by the FDA. ${ }^{9}$ This limits both safety and efficacy information. A Phase 2 study in forty-eight participants with MS tested 6 varying drug regimens. ${ }^{25}$ Twenty-eight participants $(58 \%)$ experienced infusion-related reactions, despite premedication with an antihistamine and corticosteroid. However, all were mild. A Phase 1 open-label study was conducted in five patients with acute NMOSD relapses, where participants received a single dose of ublituximab, in addition to standard of care. ${ }^{26}$ The rationale for acute treatment was based on evidence that peripheral B cells are activated during a relapse and plasmablast production of anti-AQP4 antibodies spikes, and that B cells are also found within acute NMOSD lesions, suggesting a role of $\mathrm{B}$ cells in both the CNS and peripheral blood during relapses. Three of the 5 participants experienced infusion reactions following dosing, exhibited as a transient sharp headache and body pain. Four of 5 participants achieved rapid $\mathrm{B}$ cell depletion, which remained suppressed for at least 60 days. B cells could be detected in one participant at Day 30, and repletion continued until rituximab was administered at Day 60. Two participants relapsed within 90 days, requiring corticosteroids and plasma exchange.

\section{I 9-Directed Therapy in NMOSD Inebilizumab}

In 2020, inebilizumab became the first and only medication that targets $\mathrm{B}$ cells to be approved by the FDA for use specifically in NMOSD. ${ }^{27}$ Unlike those previously discussed, inebilizumab is a glycoengineered, afucosylated $\mathrm{mAb}$ with enhanced $\mathrm{ADCC}$ activity that targets CD19-expressing $\mathrm{B}$ cells, rather than CD20. ${ }^{28}$ Because plasmablasts and some plasma cells express CD19, and do not express CD20, inebilizumab directly targets the pathogenic AQP4 antibody-producing cells. ${ }^{28}$ N-MOmentum was the multi-center, international, placebo-controlled, double-blinded pivotal trial that supported an indication for NMOSD. ${ }^{29}$ Two hundred and thirty participants (213 AQP4-Ab seropositive) were enrolled and randomized at a rate of $3: 1$ to receive inebilizumab versus placebo. The primary endpoint was time 
to NMOSD adjudicated relapse. Secondary endpoints examining differences in worsening disability, binocular low-visual acuity, MRI activity and hospitalizations between groups. The study met its primary endpoint, with a significant increase in time to relapse compared with placebo. Twenty-one (12\%) of 174 participants who received inebilizumab had a relapse during the 28 -week randomized controlled period (RCP) of the trial, compared to $22(39 \%)$ of 56 participants on placebo (hazard ratio [HR] 0.272 [95\% CI 0.150-0.496]; p<0.0001). The number needed to treat was 3.73 (95\% CI 3.06-5.66). ${ }^{29}$ The low number of AQP4-Ab seronegative participants enrolled $(n=17)$ did not provide enough data to support an indication among those without the AQP4 antibody. In the cohort of AQP4-Ab seropositive participants, 18 (11\%) of 161 participants who received inebilizumab had an attack compared to $22(42 \%)$ of 52 participants on placebo (HR 0.227 [95\% CI 0.121-0.423]; $<<0.0001$ ). The number needed to treat was 3.23 (95\% CI $2 \cdot 72$ $4 \cdot 54){ }^{29}$ Less disability worsening, fewer new active MRI lesions, and fewer disease-related hospitalizations were seen in participants receiving inebilizumab, though low-contrast bilateral visual acuity worsening was no different between groups. Following the RCP, participants were eligible to participate in the open-label period (OLP) for a minimum of two years, in which 216 participants opted in. Inebilizumab provided a sustained relapse reduction, with an ARR of 0.092 at end of study. $^{30}$

Adverse event profiles were similar between the inebilizumab and placebo groups, including infusion reactions (9 and 10\%, respectively), and none were severe. ${ }^{29}$ Like with other $\mathrm{B}$ cell depletion mAbs, they occurred more often with the initial dose. Participants received premedication with a corticosteroid, acetaminophen and diphenhydramine prior to each infusion to mitigate the risk. Infection rates and serious events were similar between groups. No safety signals emerged during the OLP, including rates of infection with increased exposure to drug over time. $^{31}$

The dosing regimen for inebilizumab was standardized in the trial: 2 paired infusions at treatment initiation, followed by a single infusion every 6 months. Infusion time is quicker with inebilizumab than it is with rituximab or ocrelizumab, such that the escalating dose is given over 90 minutes. Study data supports that this 90-minute infusion time is adhered to in most patients. ${ }^{32}$

\section{B-Cell Targeted Therapies for Myelin Oligodendrocyte Glycoprotein Disease}

In recent years, a second biomarker has been identified in a subset of AQP4-Ab seronegative patients who meet the NMOSD diagnostic criteria: antibodies to myelin oligodendrocyte glycoprotein (MOG), a protein expressed on the outer surface of the myelin sheath and oligodendrocytes. ${ }^{33}$ While the pathogenesis of this disease including the role of MOG antibodies is still being investigated, the immune system appears to be targeting MOG, a myelin protein, causing demyelination. Despite phenotypic overlap with AQP4-Ab seropositive NMOSD, MOG antibody disease (MOGAD) is a distinct immunological disease, and response to treatments differ between them. ${ }^{34}$ As of now, no drug is indicated for MOGAD, and no trials are currently enrolling for this indication. Nonetheless, one of the aforementioned trials included patients who fulfilled the NMOSD diagnostic criteria and were later found to be MOG antibody seropositive: inebilizumab in NMOSD. Of the 17 AQP4 seronegative patients included in the trial, seven were MOG antibody seropositive (6 randomized to inebilizumab, 1 randomized to placebo). ${ }^{35}$ Of the seven, one MOG antibody seropositive patient randomized to receive inebilizumab relapsed during the 28 -week RCP. These data support other findings using rituximab which suggest that, while it may reduce the risk of relapses in MOG antibody seropositive patients, B-cell depletion may not have the same degree of disease mitigation as it does with AQP4 seropositive NMOSD. ${ }^{34}$ However, controlled trials are necessary.

\section{Disclosure}

Michael Levy has received consulting income from Horizon Therapeutics (manufacturer of inebilizumab), Genentech (manufacturer of rituximab), Alexion, UCB Pharmaceuticals and Mitsubishi Pharmaceuticals. He has also received grants from these companies funding research at Massachusetts General Hospital. Maureen A. Mealy works for Horizon Therapeutics, the manufacturer of inebilizumab. Her contribution to the manuscript herein does not necessarily represent the views of Horizon Therapeutics. The authors report no other potential conflicts of interest for this work.

\section{References}

1. Mealy MA, Wingerchuk DM, Greenberg BM, Levy M. Epidemiology of neuromyelitis optica in the United States: a multicenter analysis. Arch Neurol. 2012;69(9):1176-1180. doi:10.1001/archneurol.2012.314 
2. Levy M, Wildemann B, Jarius S, et al. Immunopathogenesis of neuromyelitis optica. Adv Immunol. 2014;121:213-242. doi:10.1016/B978-0-12-800100-4.00006-4

3. Kowarik MC, Astling D, Gasperi C, et al. CNS aquaporin-4-specific B cells connect with multiple B-cell compartments in neuromyelitis optica spectrum disorder. Ann Clin Transl Neurol. 2017;4 (6):369-380. eCollection 2017 Jun. doi:10.1002/acn3.418

4. Mealy MA, Mossburg SE, Kim SH, et al. Long-term disability in neuromyelitis optica spectrum disorder with a history of myelitis is associated with age at onset, delay in diagnosis/preventive treatment, MRI lesion length and presence of symptomatic brain lesions. Mult Scler Relat Disord. 2019;28:64-68. Epub 2018 Dec 9. doi:10.1016/j. msard.2018.12.011

5. Kessler RA, Mealy MA, Levy M. Treatment of neuromyelitis optica spectrum disorder: acute, preventive, and symptomatic. Neurol Neuroimmunol Neuroinflamm. 2018;5(4):e468. eCollection 2018 Jul. doi:10.1212/NXI.0000000000000468

6. Rituxan (rituximab) [package insert]. South San Francisco, CA: Genentech, Inc.; Revised February 2010.

7. Bar-Or A, Calabresi PAJ, Arnold D, et al. Rituximab in relapsing-remitting multiple sclerosis: a 72-week, open-label, Phase I trial. Ann Neurol. 2008;63(3):395-400. doi:10.1002/ana.21363

8. Damato V, Evoli A, Iorio R. Efficacy and safety of rituximab therapy in neuromyelitis optica spectrum disorders: a systematic review and meta-analysis. JAMA Neurol. 2016;73(11):1342-1348. doi:10.1001/ jamaneurol.2016.1637

9. Ancau M, Berthele A, Hemmer B. CD20 monoclonal antibodies for the treatment of multiple sclerosis: up-to-date. Expert Opin Biol Ther. 2019;19(8):829-843. Epub 2019 May 24. doi:10.1080/ 14712598.2019.1611778

10. Kimbrough D, Fujihara K, Jacob A, et al. Treatment of neuromyelitis optica: review and recommendations. Mult Scler Relat Disord. 2012;1(4):180-187. doi:10.1016/j.msard.2012.06.002

11. Cree BA, Lamb S, Morgan K, et al. An open label study of the effects of rituximab in neuromyelitis optica. Neurology. 2005;64 (7):1270-1272. doi:10.1212/01.WNL.0000159399.81861.D5

12. Nikoo Z, Badihian S, Shaygannejad V, et al. Comparison of the efficacy of azathioprine and rituximab in neuromyelitis optica spectrum disorder: a randomized clinical trial. J Neurol. 2017;264 (9):2003-2009. Epub 2017 Aug 22. doi:10.1007/s00415-017-8590-0

13. Tahara M, Oeda T, Okada K, et al. Safety and efficacy of rituximab in neuromyelitis optica spectrum disorders (RIN-1 Study): a multicentre, randomised, double-blind, placebo-controlled trial. Lancet Neurol. 2020;19(4):298-306. Epub 2020 Mar 18. doi:10.1016/S1474-4422(20)30066-1

14. Gao F, Chai B, Gu C, et al. Effectiveness of rituximab in neuromyelitis optica: a meta-analysis. BMC Neurol. 2019;19(1):36. doi:10.1186/s12883-019-1261-2

15. Annovazzi P, Capobianco M, Moiola L, et al. Rituximab in the treatment of neuromyelitis optica: a multicentre Italian Observational Study. J Neurol. 2016;263(9):1727-1735. Epub 2016 Jun 10. doi:10.1007/s00415-016-8188-y

16. Mealy MA, Wingerchuk DM, Palace J, et al. Comparison of relapse and treatment failure rates among patients with neuromyelitis optica: multicenter study of treatment efficacy. JAMA Neurol. 2014;71 (3):324-330. doi:10.1001/jamaneurol.2013.5699

17. OCREVUS (ocrelizumab) [package insert]. South San Francisco, CA: Genentech, Inc.; March 2017.

18. Ng HS, Rosenbult CL, Tremlett H. Safety profile of ocrelizumab for the treatment of multiple sclerosis: a systematic review. Expert Opin Drug Saf. 2020;19(9):1069-1094. Epub 2020 Aug 31. doi:10.1080/ 14740338.2020.1807002

19. Patel A, Sul J, Gordon ML, et al. Progressive multifocal leukoencephalopathy in a patient with progressive multiple sclerosis treated with ocrelizumab monotherapy. JAMA Neurol. 2021;78:e210627. Online ahead of print. doi:10.1001/jamaneurol.2021.0627
20. Stamatellos VP, Siafis S, Papazisis G. Disease-modifying agents for multiple sclerosis and the risk for reporting cancer: a disproportionality analysis using US food and drug administration adverse event reporting system (FAERS) database. $\mathrm{Br} \mathrm{J}$ Clin Pharmacol. 2021. Online ahead of print. doi:10.1111/bcp.14916.

21. Caldito NG, Shirani A, Salter A, et al. Adverse event profile differences between rituximab and ocrelizumab: findings from the FDA adverse event reporting database. Mult Scler. 2021;27(7):1066-1076. Epub 2020 Aug 21. doi:10.1177/1352458520949986

22. KESIMPTA (ofatumumab) [package insert]. East Hanover, NJ: Novartis Pharmaceuticals Corporation; August 2020.

23. Hauser SL, Kappos L, Arnold DL, et al. Five years of ocrelizumab in relapsing multiple sclerosis: OPERA studies open-label extension. Neurology. 2020;95(13):e1854-e1867. Epub 2020 Jul 20. doi:10.1212/WNL.0000000000010376

24. Maillart E, Renaldo F, Papeix C, et al. Dramatic efficacy of ofatumumab in refractory pediatric-onset AQP4-IgG neuromyelitis optica spectrum disorder. Neurol Neuroimmunol Neuroinflamm. 2020;7(3):e683. Print 2020 May. doi:10.1212/NXI.0000000000000683

25. Fox E, Lovett-Racke AE, Gormley M, et al. A phase 2 multicenter study of ublituximab, a novel glycoengineered anti-CD20 monoclonal antibody, in patients with relapsing forms of multiple sclerosis. Mult Scler. 2021;27(3):420-429. Epub 2020 Apr 30. doi:10.1177/ 1352458520918375

26. Mealy MA, Levy M. A pilot safety study of ublituximab, a monoclonal antibody against CD20, in acute relapses of neuromyelitis optica spectrum disorder. Medicine (Baltimore). 2019;98(25): e15944. doi:10.1097/MD.0000000000015944

27. UPLIZNA (inebilizumab-cdon) [package insert]. Gaithersburg, MD: Viela Bio, Inc.; June 2020.

28. Chen D, Gallagher S, Monson NL, et al. Inebilizumab, a B cell-depleting anti-CD19 antibody for the treatment of autoimmune neurological diseases: insights from preclinical studies. J Clin Med. 2016;5(12):107. doi:10.3390/jcm5120107

29. Cree BAC, Bennett JL, Kim HJ, et al. Inebilizumab for the treatment of neuromyelitis optica spectrum disorder (N-MOmentum): a double-blind, randomised placebo-controlled phase 2/3 trial. Lancet. 2019;394(10206):1352-1363. Epub 2019 Sep 5. doi:10.1016/S01406736(19)31817-3

30. Cree BAC, Bennett JL, Weinshenker BG, et al. Long-term efficacy outcomes with inebilizumab treatment in neuromyelitis optica spectrum disorder: the N-MOmentum trial. Poster presented at: American Academy of Neurology Annual Meeting; April 17-21; 2021; virtual.

31. Cree BAC, Bennett JL, Weinshenker BG, et al. Long-term safety outcomes with inebilizumab treatment in neuromyelitis optica spectrum disorder: the N-MOmentum trial. Poster presented at: American Academy of Neurology Annual Meeting; April 17-21; 2021; virtual.

32. Tullman M, Ratchford J, She D. Evaluation of infusion reactions and infusion times in the N-MOmentum study of inebilizumab for NMOSD. Poster presented at: ACTRIMS Forum; February 25-27; 2021; virtual.

33. Narayan R, Simpson A, Fritsche K, et al. MOG antibody disease: a review of MOG antibody seropositive neuromyelitis optica spectrum disorder. Mult Scler Relat Disord. 2018;25:66-72. Epub 2018 Jul 24. doi:10.1016/j.msard.2018.07.025

34. Whittam DH, Cobo-Calvo A, Lopez-Chiriboga AS, et al. Treatment of MOG-IgG-associated disorder with rituximab: an international study of 121 patients. Mult Scler Relat Disord. 2020;44:102251. Epub 2020 Jun 2. doi:10.1016/j.msard.2020.102251

35. Pittock S, Paul F, Marignier R, et al. AQP4-IgG seronegative patient outcomes in the N-MOmentum trial of inebilizumab in neuromyelitis optica spectrum disorder. Poster presented at: Annual ECTRIMS Congress; September 9-12; 2020; virtual. 


\section{Publish your work in this journal}

ImmunoTargets and Therapy is an international, peer-reviewed open access journal focusing on the immunological basis of diseases, potential targets for immune based therapy and treatment protocols employed to improve patient management. Basic immunology and physiology of the immune system in health, and disease will be also covered. In addition, the journal will focus on the impact of management programs and new therapeutic agents and protocols on patient perspectives such as quality of life, adherence and satisfaction. The manuscript management system is completely online and includes a very quick and fair peer-review system, which is all easy to use. Visit http://www.dovepress.com/testimonials.php to read real quotes from published authors. 